\title{
sciendo
}

\section{How Robot/human Orchestration Can Help in an HR Department: A Case Study From a Pilot Implementation}

\author{
Dalibor ŠIMEK and Roman ŠPERKA
}

\begin{abstract}
Silesian University in Opava, School of Business Administration in Karvina, Univerzitní náměstí 1934/3, 73340 Karviná, Czech Republic, simek@opf.slu.cz, sperka@opf.slu.cz
\end{abstract}

\begin{abstract}
Background and Purpose: Motivation of this research is to explore the current trend in automating the business processes through software robots (Robotic Process Automation - RPA) and its managing within enterprise environment where most of the processes are executed by human workforce. As the RPA technology expands the demand for its coordinating grows as well. The possible solution to this challenge is shown in case study research in form of implementing orchestration platform to a concrete business process of onboarding in HR department of a multinational company. The aim of this paper is to explore the phases and activities of the pilot project implementation of Robotic Service Orchestration (RSO) in combination with RPA technology and to assess the potential benefits.

Design/Methodology/Approach: Case study research approach was selected to explore the research phenome$\mathrm{na}$, which is the implementation of RSO platform in combination with RPA technology and assessing incoming benefits. The case is formed with 2 companies - (1) multinational company with ongoing effort of automating onboarding process, (2) technology and consulting company delivering the automation solution. Data were collected through semi-structured interviews with respondents from two involved companies and by analysing internal documents.

Results: The analysis of case provided in this paper revealed some key insights: (1) strategical position of RSO and tactical position of RPA towards the existing legacy systems, (2) need for increased focus on initial process modelling phase, (3) Application Programming Interface (API) integration is more viable solution for RPA, (4) the biggest benefit of RPA - its agility, (5) future potential of the RSO replacing the BPMS.

Conclusions: First of all, there is a need of higher number of software robots adopted in a company before orchestration could pay off. On the other side, current Business Process Management Systems (BPMS) solutions don't offer functionalities for managing human and software robots workforce altogether. RPA is expected to expand and without proper orchestration the effectivity will not grow constantly.
\end{abstract}

Keywords: Robotic service orchestration, Robotic process automation, Pilot implementation, Case study, Human resources.

\section{Introduction}

Workflow management (WfM) was trending at the end of the 20th century, but as Abbott and Sarin (1994) noted, the emphasis in workflow management was on using computers to help manage business processes, which could be comprised of many individual tasks, and not on using computers to automate the individual tasks. This approach distinguishes between WfM and the recent practical trend of Robotic Process Automation (RPA). Van der Aalst et al. (2018) shared his opinion about RPA. According to his research, "RPA is an umbrella term for tools that operate on the user interface of other computer systems in the way a human would do. RPA aims to replace people by automation done in an 'outside-in' manner." This 'outside-in' manner is considered to be a great advantage, mainly in comparison with WfM. In this approach, existing informa-

Received: May 14, 2019; revised: June 28, 2019; accepted: July 30, 2019 
tion systems (IS) remain unchanged. Redesigning old IS or designing new IS is often costly in the context of an automation project as a whole. These steps are replaced by robot agents with RPA usage. RPA is a tool that adopts some profound elements from WFMSs but enhances them with the latest technology options. The practical definition provided by Gartner is as follows: "Robotic process automation tools perform 'if, then, else' statements on structured data, typically using a combination of user interface interactions, or by connecting to APIs to drive client servers, mainframes or HTML code. An RPA tool operates by mapping a process in the RPA tool language for the software 'robot' to follow, with runtime allocated to execute the script by a control dashboard." (according to Tornbohm and Dunie (2017)).

To support the statement of the trending RPA, the predictions of research companies are clear. This discipline arises from real companies' problems and the fact that they have been trying to automate routine tasks and business processes for so long, often without proper Return on Investment (ROI). The RPA market has reached US \$250 million in 2016 according to the US research company Forrester (Le Clair, 2017), and they are expecting to grow significantly with help of Artificial Intelligence (AI), which is starting to be implemented in existing RPA solutions. There are approximately 12 key vendors of RPA solutions on the market. The estimated growth provided by Forrester (Le Clair, 2017) is that the RPA market will reach US \$2.9 billion in 2021. These numbers are too large to be ignored. Academic researchers are catching up, but RPA in the academic environment is still in its infancy.

With RPA, organizations are deploying technology that can create virtual workforces of robotic workers. They are operating within the company's computational capacity to automate structured office processes. The difference opposite classic business process automation is the scope, where previous automation capabilities were there to assist the human process participants and owners. With RPA, we are dealing with potential replacement of the whole resource, which takes care of the workflow execution with no need to interrupt or redesign the background system. As Brocke et al. (2018) noted, RPA uses AI technologies to bring decision-making intelligence, flexibility and adaptability into business process environments. With this increase in AI incorporation into the processes, RPA became a significant tool in the BPM domain. Mendling et al. (2018) panel report construe the question of AI in RPA more specifically by raising question which RPA brings to the BPM research domain: "how to design and program robots and to integrate them with BPM systems, how to leverage RPA as a vehicle to support AI-enhanced processes, and how to use artificial intelligence techniques to program RPA solutions based on goals".

Human-robot cooperation (HRC), used in the research in manufacturing and assembly productions (Pellegrinelli et al., 2016; Michalos et al., 2014) has a different concep- tion, than the human/robot orchestration - the Robotic Service Orchestration (RSO) - used in this paper, perceives robots as a physical embodiment rather than intangible software. Because the BPMSs classification is quite broad, the RSO belongs into one of its categories. The purpose of RSO is similar because, according to the developers of Enate software tool, RSO is a platform that enables the delivery, management and execution of business processes that stand behind every service across both the digital and human workforce. The business logic is largely the same, but the distinction comes with the technology they are using to automate the business processes. RSO is built for close cooperation with external services such as RPA. RSO cooperates most of its functionalities from BPMSs, but it upgrades the redistribution of work among the available resources while considering humans, as well as robots. It attempts to answer a formidable question: which processes should be automated, and which should be performed by humans (Aalst et al., 2018).

With the growth of the RPA domain also comes some criticisms. As it was with Business Process Reengineering in the 1990s or BPM in the beginning of the new century, new trending technologies are very attractive to consulting or software vendor companies, which are offering a solution in the B2B market but often fail to deliver real value in terms of increasing the process effectiveness. According to Ernst \& Young report, 30-50\% (Lamberton, 2016) of initial RPA implementations fail. Nevertheless, a research report from Hindle et al. (2017), provides data about different aspects of RPA implementation (specifically the Blue Prism software tool) based on a survey research strategy. This report (Hindle et al., 2017) shows that except for one case, every other case (23) had positive ROI. This contradiction shows how this rapidly growing industry is unstable and unclear. This circumstance, of course, provides a great opportunity for researchers to uncover the vail of uncertainty and bring valid conclusions into this newly formed domain.

The aim of this paper is to explore the phases and activities of the pilot project implementation of RSO in combination with RPA technology and to assess the potential benefits. The subject of the research is the whole implementation process, which consists of different phases and activities sorted on a time scale. Two companies are cooperating on showcasing the benefits of RSO and RPA technology with the aim of improving the process of onboarding (HR department). The research questions support the aim of the paper, as follows:

What was the process of the implementation of the examined project?

What are the benefits for companies A and B that arise from the examined project?

According to our knowledge, this type of a case study, 
with implementation of BPO and RPA, has never been covered in academic journals. Thus, this research area is a white space in the literature, and only the research process will demonstrate how this particular research design suits the new wave of approaching process improvement and automation. This circumstance often occurs in exploratory research projects.

As is usual with a new stream of research, the first exploratory studies in the field are necessary for achieving in-depth insights, which is the reason why we choose exploratory study, and the subsequent content is structured accordingly. After the related work section, a methodology section is offered where an appropriate research design is described. Next, we present an actual case study that is subsequently structured as the project (case). A discussion followed by conclusion is provided at the end of this paper.

\section{Related work}

Recently, papers on RPA have started to emerge. Most of them are presented in the form of a case study, such as (Fernandez and Aman, 2018; Aguirre and Rodriguez, 2018; Lacity et al., 2015a; Lacity et al., 2015b; Lacity and Willcocks, 2016). In research from Lacity et al. (2015), single case studies are presented. They investigate real examples of practical usage of RPA in companies such as $\mathrm{O} 2^{1}$. In the case of $\mathrm{O} 2,2$ pilot processes were selected, and the results show higher ROI in comparison with BPMS implementation. Other interesting results are displayed in the Aguirre and Rodriguez (2018) case of implementing RPA into a business process outsourcing company, where an increase in productivity and capacity of approximately $20 \%$ was reported.

From a methodological standpoint, researchers should be aware of the overall methodological selection and preparation of case studies. There is a need to distinguish between the case study, which is commonly presented as marketing material from software providers and consulting companies, and case study research (Saunders et al., 2016). The most significant paper is that of Fernandez and Aman (2018), because it is the only paper that outlines the research design and methodological selection. They also used a single case study, and data were collected through semi-structured interviews with various respondents, mostly process participants. They conducted 11 interviews, and the results from those interviews are presented, including the impact on individuals and the company context.

The most positive results are shown in the case studies from Lacity et al. (2015) and Lacity and Willcocks (2017), where the subjects were the companies Xchanging and UTILITY (anonymized name). In the first case of Xchanging, the results exceeded the first expectations. Overall, 14 key processes were automated with a help of 27 implemented robots. They processed 120 thousand instances per month with an average savings of $30 \%$ on every automated process. The UTILITY case was even larger, with 25 processes involved in the RPA initiative and with 1 million instances per month. This amount of work is performed by 300 robots, which are orchestrated with 2 employees, and they substitute for the work of 600 people. The ROI from this project is $200 \%$ for the first year after the implementation. The overall return on investment with the RPA project mentioned in the work of Lacity and Willcocks (2016) is typically 1 year.

In the most recent work from 2019 is clear that the RPA technology is starting to engage with diverse industries. The research paper from Houy et. al. (2019) demonstrates an example of implementing cognitive RPA to public administration, Moffitt (2018) outlined how audit could benefit from use of RPA and another research is testing RPA in digital forensics (Asquith and Horsman 2019). Also another case studies from more traditional industries are still emerging. For example Schmitz et al. (2019) described case of German telecommunications operator where RPA was use as an enabler to realize digital strategy. The RPA is not used only in new areas, but with help of AI, to a new strategic business task such as decision-making (Ranerup, Henriksen 2019).

\section{Research methodology}

The aim of this section is to construct research questions and to propose a research design that will serve to answer the research questions. The research design guides the investigator in the process of collecting, analysing and interpreting the observations during the case (Yin 2014).

To better understand this methodology section, a case definition is presented first. The definition specifies the scope of the case. In this study, a case is a pilot project implementation, which involves two companies in a specific time horizon. The first company is developing and providing an RSO and RPA solution (company A) to another company, which is a multinational company that operates in a business process service market (company B). Company A is a start-up company, which was founded in 2014 in the Czech Republic and currently has 12 employees. Company B is an international enterprise founded in 2004 in the Czech Republic that specializes in providing business processes and services for large corporations across the globe, with approximately 1600 employees.

Company A operates in the RPA market and has a strong background in data science and text mining. They already cooperate with company B on a project on the automation of a certain task through the deployment of an

$1 \mathrm{https} / /$ www.telefonica.com/en/home 
RPA solution. Given the fact that Company B is operating on a business process services market, they are embracing new automation technology, which company A is deploying. Their cooperation creates a mutually beneficial synergic effect. The case in this case study research is a pilot project of implementing RSO and RPA in the HR department of company $\mathrm{B}$, in particular, an onboarding process. The implementation is led by company A.

The methodological choice of this paper reflects the fresh essence of this domain. That is precisely why we decided on an exploratory study in our research. A small sample and deep dive into the research phenomenon are characteristics of a qualitative exploratory study. As a prime research technique, unstructured interviews were chosen.

It is necessary to define the purpose and scope of the case study. As Schramm (1971) noted, the essence of a case study and the central tendency among all types of case studies is that it attempts to illuminate a decision or set of decisions - why they were taken, how they were implemented, and with what result. This illumination comes from examining the contextual conditions, believing that they might be highly pertinent to the phenomenon of study (Yin, 2014). According to Feagin et al. (1991), case studies concern decisions, programmes, implementation processes and organizational change. The case study in this paper concerns implementation processes.

Only one case was chosen, and thus, it is single case study research, which is common for exploratory studies. A rationale justification for building this paper on only one case comes from Yin (2014). He states that the reasoning for single case study research comes from a situation in which the case represents an extreme or unique case, such as conducting a pilot project.

The units of analysis are the individuals who are part of this project and the documents, which provide data about the course of the project and about the deployed technology. Case boundaries are set by the pilot project, which determines everything directly related to the implementation process: its content, participants, procedures, logical structure and IT technology. On the other side, everything outside is context - the ambient conditions that influence the project as well. Another boundary is the time frame of this case: the beginning is when both sides (companies A and B) kick-off the first idea of this pilot project, and the end is when the deployment was evaluated in the form of a report with a follow-up meeting.

To secure the construct validity in the research design, Yin (2014) recommends using multiple sources of evidence, which in our case study are narratives from different participants (from both company A and company B), project documentation and other documents (about the BPO and RPA solution).

To obtain access to the data within these companies, the authors had to negotiate first. They were involved in the project during its realization, and they signed a nondis- closure agreement with company $\mathrm{B}$, which has strict rules for research within its span.

One unit of analysis is formed by the technique for collecting data - semi-structured interviews. They were conducted in the first half of 2018 and form the main source of data for this case study. Access to four respondent (Table 1) narratives was negotiated, and the methodological preparation for these interviews was completed. Clear boundaries for the interview were set for the pilot project. Each interview took approximately 2 hours. This span is the average length for in-depth exploratory interviews, and they were recorded for consequent transcription. First, the respondents were acquainted with the flow of the interview and with moral and ethical concerns. After this acknowledgement, the respondents were invited to interpret their narrative onto this pilot project. Next, additional questions were asked to gain supplementary information, which provided thoughts that emerged from a further narrative. Other questions addressed the information that shapes the broad illustration of the context that surrounds the case. These questions were split into a few groups, each of which addressed different aspects:

- Describing the motivation for the examined project

- Describing the phases and activities in the pilot project

- Addressing success in these different phases

- Evaluating the benefits that resulted from the examined project

Table 1: Summary of Respondents

\begin{tabular}{|c|c|c|c|}
\hline $\begin{array}{l}\text { Respondent } \\
\text { number }\end{array}$ & $\begin{array}{l}\text { From } \\
\text { company }\end{array}$ & $\begin{array}{l}\text { Position within } \\
\text { the project }\end{array}$ & $\begin{array}{l}\text { Length of } \\
\text { the interview }\end{array}$ \\
\hline 1 & A & solution designer & 1,5 hours \\
\hline 2 & A & RPA Developer & 2 hours \\
\hline 3 & A & CEO & 2 hours \\
\hline 4 & B & process owner & 1,5 hours \\
\hline
\end{tabular}

The composition of the respondents can be seen in Table 1. Three are from company A, and one is from company B. This aspect is caused mainly by the fact that most of the work in the project was performed by company A representatives and that this project was taken as a pilot to validate the new technology for optional launch and further implementation projects. These interviews provide authors with the main source of evidence, but the conclusions cannot be based entirely on interviews. For this reason, the authors asked for documents from software providers (supplied with software instructions and training materials) and project documentation (including process models) held by company A in an unstructured format, but supported with tutorial videos. After the interviews, addi- 
tional conversations via email were engaged because, as Yin (2014) noted, case study data collection is not merely a matter of recording data in a mechanical fashion. You must be able to interpret the information as it is being collected and to know immediately, for example, if several sources of information contradict one another and lead to the need for additional evidence. This assertion is aligned with the authors' interpretative research philosophy.

As Yin (2014) noted, critics often discuss the subjective manner in which judgements are used to collect the data in case study research. The same circumstance occurs for generating interpretations in the research philosophy, since its purpose is to understand and not necessarily to measure a phenomenon (Saunders et al., 2016). For this reason, we discuss the methodology very broadly to ensure the validity and reliability, which will preventively serve to eliminate errors and biases in the study.

\section{Pilot implementation of RSO and RPA}

A brief introduction of the company involved in the project was given in the previous section, but to provide broader information, the specific software used in this case must be introduced. One product is the RSO software platform called Enate. The next important technologies in this case are two RPA tools - Blue Prism and UiPath. There are additional systems (HR system Target, K2 BPMS) that were incorporated into the process within this cooperation, but they did not represent the main units of analysis, and thus their introduction is not needed.

The internal training materials provided to company A by Enate allow an operations manager to remain in control of "who does what", even when the work is being performed by robots. In other words, Enate is a platform where the workflow is created and human workers, RPA robots or other digital agents execute activities within this workflow, which comprises the end-to-end service. Additionally, a console for monitoring and measurement is provided by Enate. Process owners can manually intervene when pertinent to override the standard business rules that are being used by the system to prioritize and distribute the work. Another large benefit in the context of this case is that the operations manager can decide which robots are assigned to individual work queues. Thus, multiple RPA solutions can be integrated to draw coherent benefits. This integration is accomplished through API. As the CEO of company A said in an interview "Enate is a workflow management system (WfMS) with the function to integrate and thus manage robots as well as the human workforce."

Both UiPath and Blue Prism work within the premise of RPA. According to the Forrester report (Le Clair, 2017), UiPath, Blue Prism and AutomationAnywhere are the top 3 RPA vendors. UiPath uses Microsoft's Workflow Foundation in its design studio, and it relies on external partners for direct implementation. UiPath's advantages come out form an open platform and in the creation of their global community, which serves as a home for RPA developers. On the other hand, Blue Prism provides strong load balancing, restart functionality, encryption at rest, audits, and desktop-aligned robots, which are defined and managed centrally. Blue Prism does not have open access to training materials, and it is harder for users to learn and explore its capabilities.

Next, we outline the motivation component, where the summary of reasons to participate from both companies is presented. Subsequently, the concrete flow of activities is described, and in the end, the evaluation of the project is showcased. The next part is already interpreting the interviews as a source of primary data.

\subsection{Motivation from both companies to participate}

Because the scope of this pilot project implementation is large and its potential is far-reaching in terms of investments and costs, which are primarily significant for company A, there should be a strong motivation toward this case. This stands at the very beginning of applying the new approach and sparks its initiation. This section unveils the initial expectations, which are to be further compared with actual outcomes.

The CEO of company A attended an international conference where he met the CEO of Enate, who has a strong opinion about his product, which was aligned with how he was thinking about the future for RPA and BPM as a whole. The CEO of company A said "I see in the last 2-3 years a tendency of enterprises to pull back from traditional tools, which are in most cases burned out and already fully used on behalf of process change. Therefore, they already have their processes straightened, but now they are facing an unpleasant situation with inhouse systems and with their centralization or transition towards one system, which is tedious and complicated. That's the reason why RPA has a chance to catch their attention." His statement is supplemented with a review in the introduction of this paper, where one of the main benefits of RPA is that it is built upon existing systems. The outcome from this part of the interview is that this benefit is the number one reason why RPA attracted a large number of enterprises.

As he continued, he explained the role of RSO: "The tendency of RPA is reaching enterprises not only in Western Europe but also Central and Eastern Europe, and this trend will continue. Enterprises will continue to invest in increasing robot capacities, gaining more robot instances and of course combining robots and the human workforce. In this stage, there is a room for manager or orchestrator, who will have the ambition and power to manage the task in real time, organize working queues, manage robot utilization and coordinate how transfer between robot/human or human/robot works. This kind of orchestration will have 
significant importance in the future." This statement also concurs with researchers in the BPM domain, who already see this shift, and as Aalst (2018) noted, the larger question is which processes should be automated and which should be performed by humans? RSO has the ambition and potential to answer this question. This point was the beginning, where company A started to think about initiating the orchestration of a project. Another reason was that they wanted to expand their portfolio of RPA tools from Blue Prism to another platform - UiPath - to be able to leverage advantages for different opportunities.

On the other side, motivation to start this cooperation from company B is built on the ongoing initiative, which has been already running since 2017 and has an ambition for improving 4 large processes in the HR department of company B:

- Onboarding

- Change in labour-law relationship

- Offboarding

- Managing maternity/parental leave

According to the owner of the onboarding process, who is also incorporated in this initiative: "These 4 processes are administratively very demanding, and activities within them are repetitive, which offers great potential for their standardization and automatization." This initiative starts with the onboarding process, where migration to the K2 BPM system was initiated to manage the whole process under one system. During the implementation, they ran into a problem, where data from the K2 system to the HR system Target had to be imported. While solving this problem, they encountered RPA, which struck them as a "great solution" according to the onboarding process owner. That is how the cooperation between the companies involved in this case study started.

Choosing the right partner for company $\mathrm{A}$ in this pilot project was clear because of the good relationship between the two companies and because of the RPA implementation, which was already in place at company B. Another reason for testing the RSO comes from the initiative at company B (described earlier), where the implementation of additional robots is planned for future projects. Thus, the $\mathrm{CEO}$ from company A and the HR process owner from company B started to negotiate the conditions and course of the pilot orchestration project. According to the solution designer, who also attended this negotiation, the aim of the project was "to improve the business process."

\subsection{Process discovery and building an as-is process model}

After approval from both sides, an appropriate process must be selected. Given the fact that the RPA robot is already incorporated into one process, while company $\mathrm{B}$ is under an ongoing change initiative in the HR department, the onboarding process within the HR department was chosen as an ideal candidate for this pilot project. Parts of this process are highly structured. Two core systems and two external services operate within the process, and before the RPA implementation, a large amount of manual work (data manipulation) must be done. As Fersht and Slaby (2012) noted, these are the very fundamental characteristics for the process to be the right candidate for RPA implementation. The proper selection for RSO subjects uses the same criteria as suitable RPA candidates, with the exception that it is advantageous if the process has handovers between human and robot workers throughout the flow. That is correct in the case of onboarding processes, where a large number of personal contacts with the applicant is required. When the process for the project was set, working groups were organized on both sides. On the company B side, an onboarding process owner and a project manager supported the owner from the IT standpoint. On the company A side, a group around the main solution designer had the RPA developer at their disposal with the support of the CEO of company A.

Company B did not have the initial workflow model according to the CEO of company $\mathrm{A}$, but in the interviews, the process owner from company B said that they already had "some" model that served as the main input for building an as-is process model. This contradiction only shows insufficient terminology alignment, which could cause further problems in a project. Thus, according to team members from company A, the first phase led to process discovery, which finished with as-is process. The discovery was based on several interviews with process owners and process participants and on process documentation from company B. After a few weeks, the workflow model was built and validated.

Company B operates with $\mathrm{K} 2$ software, which is presenting itself as a BPM system. In the workflow, the map is represented in green activities. Another system entering into workflow model is the so-called portal, which runs under the K2 system. It is a cloud solution, where applicants have an access point and can communicate through it. It is symbolized by red colour activities. Finally, yellow activities represent email actions, and one blue activity (entering data into the HR system), Target, represents the HR system. 

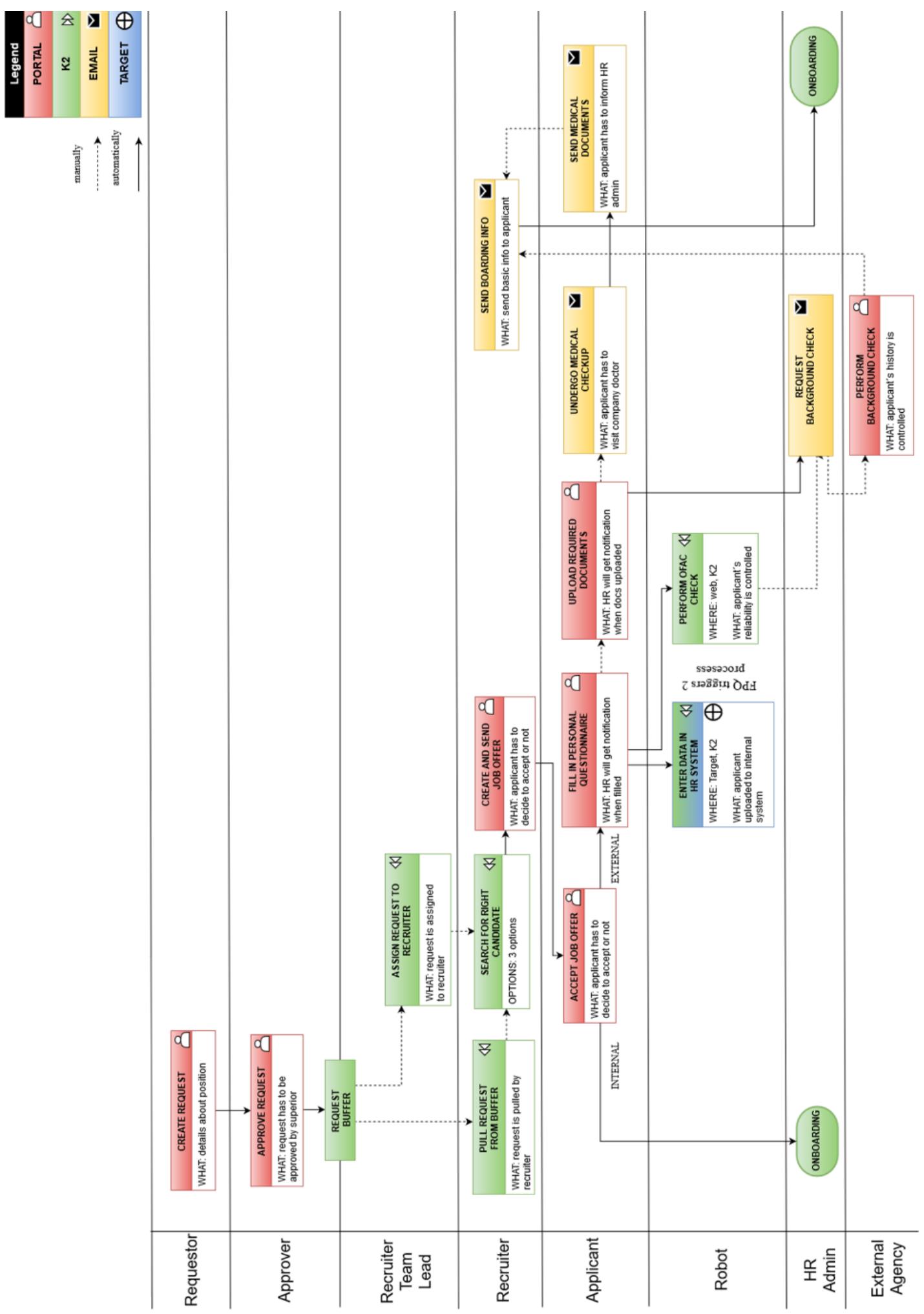

Figure 1: As-is process model. Source: internal documents of Company A 


\subsubsection{As-is process description}

The as-is process description was put together by analysing a transcript of narratives of representatives from both companies which was done by project team from company A. Final process model is shown in figure 1. It should clarify the onboarding process itself, which is a prerequisite for grasping the whole implementation project. The onboarding process starts with a requestor creating a request that contains details about the position (job description). This requestor is typically a team leader who has the competency to raise a request about the new job. This step must be approved in the internal portal by an approver, and then it routes to the request buffer in the K2 BPM system. From this buffer, the individual cases are pulled from the queue by recruiters or are directly assigned to some of the recruiters. Then, the actual selection of the right candidate is performed through a series of interviews - one phone interview and then two rounds of recruitment procedure. From this step, the successful candidate is chosen, and at the same time, a job offer is generated, and a recruiter then determines if the candidate will be onboarded. After this step, there are two options. If the applicant is (1) an internal employee, the process is now finished because he/ she had already been through the following steps. If it is (2) an external applicant, then a series of further actions is necessary. After filling out the personal questionnaire in the portal, the RPA robot (Blue Prism) performs 2 tasks (Figure 2). The first task is to enter data from the $\mathrm{K} 2$ portal into the HR system in Target, and the second task is the OFAC (Office of Foreign Assets Control) check, which is done through a publicly available database on the internet and ensures foreign verifiability in terms of terrorism, financial crimes, and so on. Hereafter, the applicant is requested to upload documents such as a certificate confirming their education, personal photo and, depending on the position, specific security documents. This step is not dependent on tasks performed by a robot, but the series of subsequent tasks already are. These steps assure a medical check-up for the applicant and that the document will head back to the recruiter. In combination with a successful background check performed by the external agency, the HR admin finishes this process with complete onboarding. The onboarding process frequency is approximately $30-50$ instances per month.

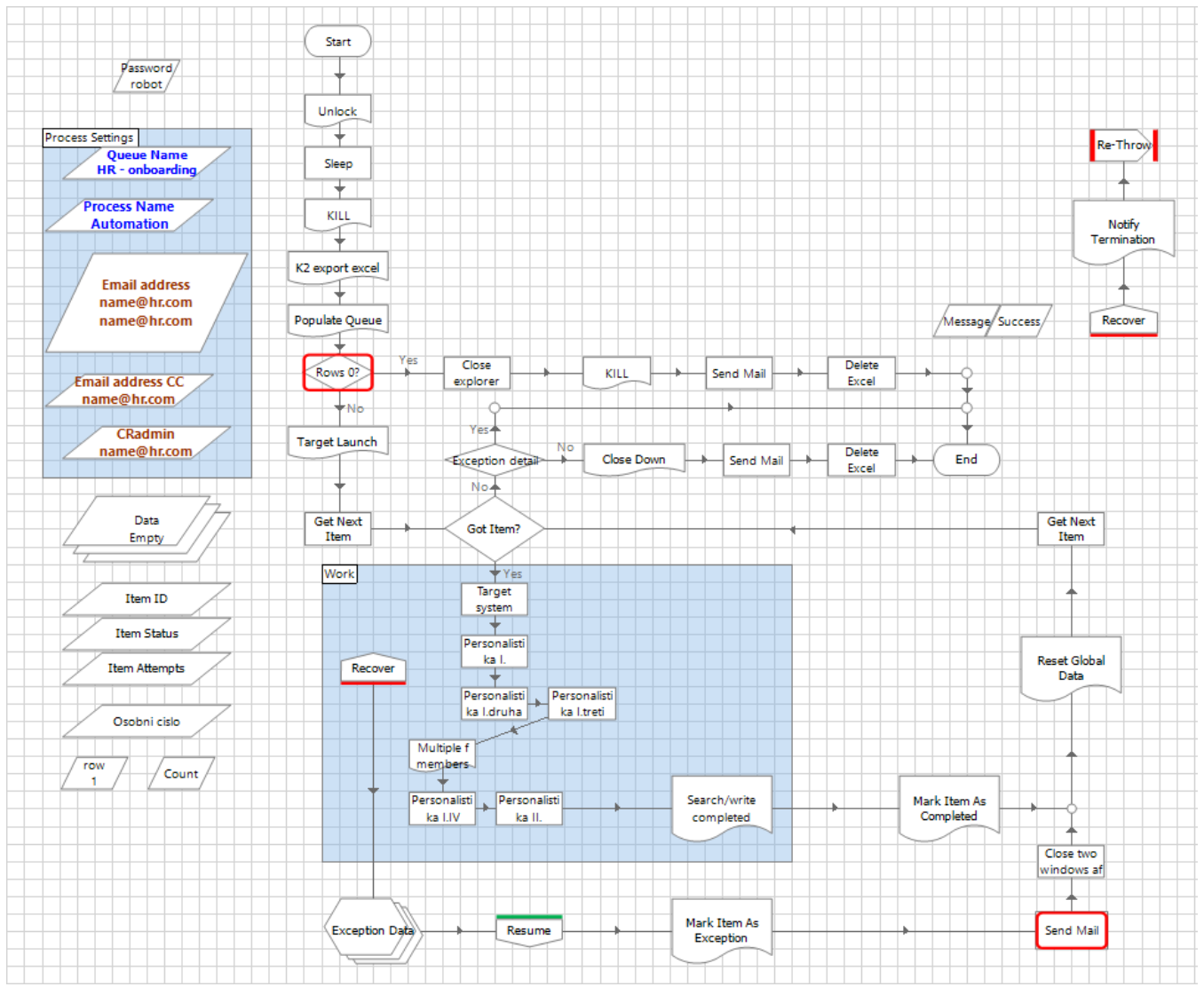

Figure 2: Illustration of part of the workflow from Blue Prism, representing the "enter data in HR system" task. Source: Internal documents from company $A$ 


\subsection{Process discovery and building an as-is process model}

Simultaneously with the process discovery phase, the training in the RSO took place in company A. They already obtained training in the RPA because that is the core business of company A, but RSO and Enate software were completely new for them. There were 3 levels of training. A total of 3 developers entered training level 1, which was more generally focused on an introduction to the Enate tool from the end-user viewpoint. Level 2 training continued as a gradual to-be process model was built. Only one RPA developer advanced to this level. Training in this phase was based on individual appointments via online calls to discuss the progress on the case. The content of this training was formed around the developers' issues, such as the connectivity to RPA platforms. Right after the first level of training, the RPA developer and solution designer from company A started to build the to-be process model in Enate, which was to experiment with all of the tool capabilities. Training in the RPA implementation was not necessary because the RPA developer and solution designer were already trained in the Blue Prism and UiPath software.

\subsection{Designing the to-be process model and implementation}

As a solution designer said: "In order to succeed with this project, we needed to consult the support from Enate iteratively within weekly cycles because this type of project was a pilot even for them". The solution designer meant that even Enate did not integrate these two (Blue Prism, UiPath) RPA platforms before. The reason is the new essence of RSO tools such as Enate. This support from the solution provider turned out to be crucial. The aim of this phase was to transform the as-is model to the to-be process model in Enate and to secure its functionality. First, the overall process logic had to be created in Enate. The complexity of the as-is model was altered by a simple 5 phase workflow. To better understand the logic of Enate, the basic archetype definition from the Enate training materials is required. First, there is a case, which represents one process instance and a series of follow up steps. On the same level as a case, there is a ticket (used for do-done diagrams). The case is split into steps, and steps are further divided into actions. The initial reflection was that this process will be composed of several cases, but after the feedback from Enate's designers, they decided that the whole process will be classified as one case to simplify the process logic. As was previously stated in the motivation section of the paper, one of the incentives was to widen the portfolio of provided RPA solutions by company A. To fully exhibit and discover the potential of Enate, the main idea was that two RPA platforms (Blue Prism, already engaged in the as-is process, and UiPath) will be deployed to this process - each of them integrated within Enate. "We can talk about real human/robot orchestration only by providing this solution", the solution designer noted.

When the overall logic was set, there was time for RPA integration. The RPA developer proclaimed: "We used the API interface working on REST services for integrating both robots from UiPath and Blue Prism. The original solutions were that robots will work just like a human, even on this interface, which could cause problems. However, our final solution was that the robot was not working on the Enate interface as a human worker, but instead entered orders through the web service." Robots were working based on the pre-defined scheduler because of RPA robots interacting with the pull from the queue command, which serves as a check on whether there is some instance in the queue waiting for a robot. There are 3 types of working items in Enate:

- Manual type action

- Email action

- Actions that could be performed by a robot - run the robot task

\subsubsection{To-be process description}

The description was obtained from the internal documents (project video) of company A. The to-be process model itself is showcased in figure 3. First, part of this phase was to determine the potential for process redesign and enables the process to enter the next step in the best manner possible. The process redesign merged the first 2 steps of the process because there were 2 non-value adding steps to create and approve the new request, and they were redesigned into a request validation step. This redesign is rather cosmetic, but it provides simplification for the process model, which is now more definite. By opening a new position, the new case (work packet) in Enate is created. The output from this initial step is assigned to a specific recruiter. Further actions are dedicated to selecting the right candidate, and after the selection, the right candidate's profile is created in Enate by the recruiter. Next, it is time for processing all of the necessary documents and data about the candidate. These actions are performed by the RPA robots - each of them (Blue Prism, UiPath) performing different tasks. The UiPath robot now performs the OFAC check previously done by Blue Prism, and the Blue Prism robot retains the execution of administrating HR related data in the Target HR system. All of these steps are performed in real time and are controlled by Enate. When both of the robots are finished, the Enate automation capacities come into play. Enate generates a new email to the applicant with medical check instructions attached, and the system sends out the information automatically. When the medical check-up is completed, a new email is generated with on-boarding information. After this step, the candidate is ready to be on-boarded, the case is closed, and a full history of action is provided and available. 


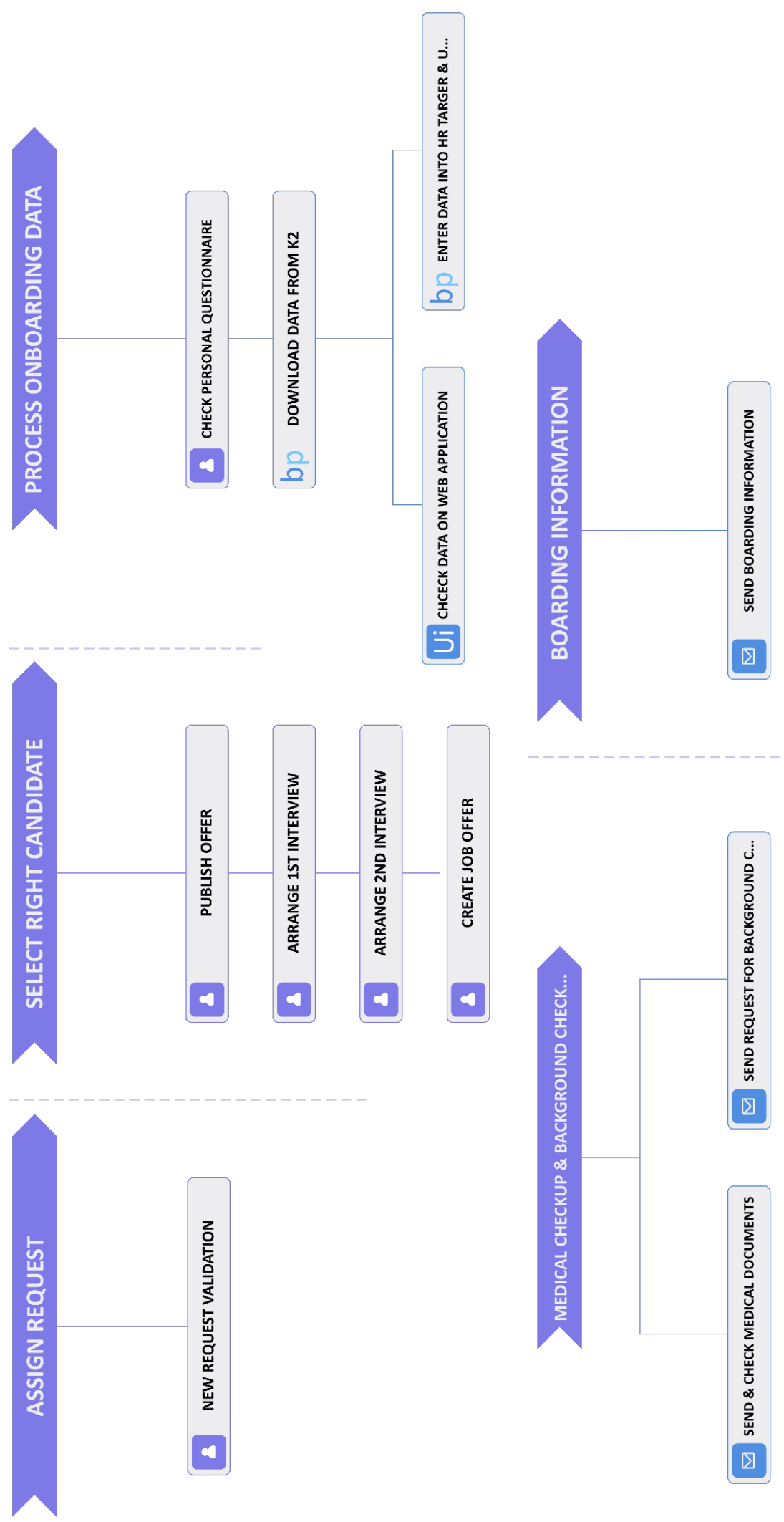

Figure 3: To-be process model designed in Enate 


\subsection{Testing and Validation}

To validate the proposed solution, a series of tests must be done to secure the proper functionalities. According to the solution designer, the testing was logically split into two phases. Separate testing is performed for the RPA robots and - then for the RSO platform - Enate. In the case of RPA robots, according to the RPA developer: "Testing was done in close cooperation with company $\mathrm{B}$, where we obtain an anonymous dataset that we ran through the workflow." This is an ordinary procedure, which focuses on the part of the workflow where a human worker makes many mistakes. From this part of the testing exception handling models are created and built into the original workflow. Company B established the test environment in the final destination - Target system. The RPA developer then could set up the robot on the real interface without potentially causing issues in the real-time version. After the exception handling models are created and the data test shows no more exceptions arising, the access to the real-time version of Target was managed. Under the tight supervision of HR administrator, the first real workflow was done through robots and then was checked to confirm the correctness of the embedded data. This test was conducted several times, and then the robot went fully into life. The overall time requirement for testing the RPA robots was the same as the time intended for robot developing, and thus, if one robot took 10 hours to construct, an additional 10 hours was the approximate time required for the testing. The follow-up testing continued onto the RSO layer. Here, the integration of robots was tested to validate the ability of Enate to communicate with both RPA platforms - Blue Prism and UiPath.

\subsection{Innovation and benefits (evaluation)}

The original agreement between companies A and B did not account for advancing the project into the production phase - live operations. The main purpose of this pilot project was to show the potential and test the new platform (Enate) in real conditions and to prove technical feasibility, much as it is in proof-of-concept scenarios. Outputs from this pilot project will be used in the future when company $\mathrm{B}$ is planning to scale robot capacities within the broad HR initiative, as was mentioned in the motivation section. The RPA developer noted: "To achieve real results, there is a need to scale the robots, which will proportionally increase the value brought by the RSO." This claim is supported by the HR process owner from company B: "In the context of our whole initiative in HR, we are talking about hundreds of cases per month, where several robots will participate. When this happens, Enate will run above K2, and other systems and managers will have a global view on the human/robot execution of processes."

The evaluation went on immediately after testing and validation. According to the CEO of company $\mathrm{A}$, the evaluation was split into two groups, namely, internal (company A) and external (company B) factors (Table 2). Internal here means the amount of time resources dedicated towards this project in order to assess it on behalf of similar future projects. Quantification was measured in Man Days (MD), which represents one working day (8 hours) of one employee. According to the RPA developer of the company A, this whole pilot project took $28 \mathrm{MD}$ : $5 \mathrm{MD}$ for the process analysis, $13 \mathrm{MD}$ for RPA development and 10 for RSO implementation.

The external factors are further split into soft and hard benefits. As the CEO of A company said, "The hard metrics remain the same as usual - different sets of key performance indicators (KPIs), processing time, quality, throughput, resource utilization or bottleneck identification." A boost to all of these metrics could be delivered by RPA and RSO, as seen in the presented case. According to the process owner of company B, the improvements in partial activities performed by robots are astonishing. The processing time of the first robotic workflow - entering data into the HR system (Target), was reduced from 15 minutes (done before by humans) to 3 minutes (done now by a robot), without any intervention needed. The OFAC check workflow time was reduced from 5 minutes to 1 minute. Based on the reported average number of cases per month (30-50), the overall savings from these two workflows are 10 hours per month, which could be recalculated to 1,25 MD saved for every month. In the context of the entire onboarding process, these savings did not cause much improvement in the processing time because the process is too complex. It still takes several days or weeks to be completed and relies on the participation of external subjects such as applicants, external companies, medical check-ups, job interviews, and so on. However, the most important qualitative benefit according to the process owner and solution designer is rooted in the reduction of error rates, in which the robots contribute close to zero errors.

Another large benefit arises from the soft metrics. The CEO of Company A shares thoughts on this topic: "Our company could use this robotic capacity when human resources are over-utilized or when the HR department can't manage to onboard or train on time. Another huge field of opportunity is the highly repetitive tasks, which are still done by humans and could cause burnout and other professional issues. This could be undertaken by robot capabilities, and in a time where work-life balance is a trend, it could be communicated as an employee benefit." Next, advantages are provided to the HR managers, who can organize work in real time and obtain reports with analytics. The RPA developer mentioned additional value for the process participants, who execute the workflow, as it is an opportunity to obtain support when guidance through the process is needed. Then, the participant could ask directly for support through Enate to the IT department or colleague. The perspective of the process owner from compa- 
Table 2: Summary of quantified evaluation

\begin{tabular}{|c|c|c|c|c|}
\hline \multicolumn{2}{|l|}{ Company A } & \multicolumn{3}{l|}{ Company B } \\
\hline Activities & $\begin{array}{c}\text { Time con- } \\
\text { sumption }\end{array}$ & Task & $\begin{array}{c}\text { Done } \\
\text { by } \\
\text { human }\end{array}$ & $\begin{array}{c}\text { Done } \\
\text { by } \\
\text { robot }\end{array}$ \\
\hline $\begin{array}{c}\text { Process } \\
\text { analysis }\end{array}$ & 5 MDs & $\begin{array}{c}\text { Enter data } \\
\text { from K2 } \\
\text { to Target }\end{array}$ & 15 min. & 3 min. \\
\hline $\begin{array}{c}\text { Develop- } \\
\text { ing UiPath }\end{array}$ & $3 \mathrm{MDs}$ & $\begin{array}{c}\text { Perform } \\
\text { the OFAC } \\
\text { check }\end{array}$ & 5 min. & 1 min. \\
\hline $\begin{array}{c}\text { Develop- } \\
\text { ing Blue } \\
\text { Prism }\end{array}$ & $10 \mathrm{MDs}$ & $\begin{array}{c}\text { Number } \\
\text { of cases } \\
\text { per month }\end{array}$ & \multicolumn{2}{|c|}{$30-50$ cases } \\
\hline $\begin{array}{c}\text { Develop- } \\
\text { ing Enate }\end{array}$ & $10 \mathrm{MDs}$ & $\begin{array}{c}\text { Savings } \\
1.25 \mathrm{MDs} / \mathrm{per} \\
\text { month }\end{array}$ \\
\hline Sum & $28 \mathrm{MDs}$ & & \multicolumn{3}{|c|}{} \\
\hline
\end{tabular}

ny B is rather pragmatic. She said that with the shortening of the processing time of key activities by transferring the workload to robots within onboarding process, there is additional space for recruiters to take care of applicants and to serve them more quickly, which is a competitive advantage in the labour market. Simplification of process management is achieved as well. The HR process owner claims that "I didn't have to go through several systems to manage the process. I will have everything organized on one dashboard." This feature is a great benefit because it saves time for the manager, who is considered to be a more expensive resource in the company.

The CEO of company A speaks about the innovation in the approach taken in this project as one of the first of its type, when both human and robot workforces are working together under one auspice. He explained the situation by saying: "If we use BPMSs, we can manage human resources and through this platform see everything we need except for the work done by robots. On the other hand, RPA provides us with analytics and dashboards to support our decision making, but only on the robotic part. Enate is standing above both these systems to provide managers with a complex picture of robots and humans working together." The interview shows that respondents perceive RPA as a main provider of quantitative benefits (improvement in the processing time) and RSO as a technology that brings qualitative benefits (better management and quality). Even though the last part of the case study is presenting an evaluation, it is meant to strengthen the exploratory findings. The authors consider the evaluation of such a unique case important because it will affect the future potential of this type of technology.

\section{Discussion}

The overall case study brings insight into the implementation of orchestration solution. This could inspire other companies which are considering usage of RPA or RSO technology and provide them with exemplary case outlying the course of implementation project. The theoretical implications are predetermined by exploratory nature of this study which helps authors deeply analyse this trendy technology and grasp for the future research. It also uncovers the future potential for this technology and showcase its strengths.

The limitations of this study are rooted in single case study research, where every outcome arises from the one particular case. Even though this case is a very specific and unique case, it draws the additional question of how these outcomes are replicable and useful for future research. The authors consider the pilot nature of the project to be a limitation because it could cause divergence in behaviour in comparison with the fact that the involved participants will anticipate that this project will transfer to live operations. These questions can only be answered by further research because of the RPA and the predominant RSO frame are white space in the BPM community. Another limitation of this study is the number of respondents from company B. Authors interviewed only one respondent from company $\mathrm{B}$, which limits the conclusions.

For future research, the authors intend to work with the premise that RPA and RSO are changing the BPM domain from the standpoint of implementation/deployment approaches and/or critical success factors. Authors intend to create a valid framework for implementing RPA as an effective automation tool. It should help companies adapt to even more agile approach of automating business processes. Additionally, with increases in the numbers of robots implemented and growing RPA initiatives in companies such as company $\mathrm{B}$, the need for robot/human orchestration will increase. Hence, multiple case study research or research based on larger samples is required, which could bring these assumptions to a more reliable and generalizable form.

RSO expansion is according to CEO of company A not matter of present. Companies are occupied with digital transformation and implementing RPA, so RSO will come after RPA achieves higher level of maturity, which will lead to broader adoption. Authors predict that human/ robot orchestration will be an emerging topic in BPM community.

In the interview, the solution designer stumbled upon the forecast for the RSO. "The development of IT capacity and overall technology will move forward to a state where robots will not only execute the workflow but also assign the work across the company to both robots and humans. This step needs considerable development of AI and self-learning algorithms." Another associated topic is 
a question of ethics and morale, because the robot in this forecast serves more as a manager. This shift is considered across society and is a controversial theme for discussion, but as was said by the CEO of company $\mathrm{A}$ in the evaluation section, robots are made for serving humans in automating highly repetitive tasks, which are often quite unpleasant and implacable for human workers. Another interesting topic of future research could be how internally communicate changes in ration of human-to-software robots workforce.

\section{Conclusion}

Our research paper addresses the current trend in the field of automation of business processes among consulting and technology companies - RPA, and adding an orchestration layer assured by the RSO system Enate.

In presented exploratory case study, the main focus was to explore the driving forces, the variables and the overall approach, which are represented by a set of consecutive phases and activities in implementing the pilot project

To distill the descriptive form of case study to the usable ideas which are signature for exploratory studies, authors extract these key insights:

- The implementation phase shows that the integration through API is a more reliable solution as using screen scraping or screen recordings techniques. When using screen scraping, the RPA robots are depending on the stability of underlying IS.

- The BPM maturity or previous experience is not needed for RPA as it is ,only“ the automation tool. On the other hand, in terms of RSO, BPM maturity is needed. RSO have to build on existing process architecture or outgoing process initiative. RPA is perceived by respondents as a tactical tool but RSO is already implemented in the strategy level of management.

- $\quad$ RSO is concurrent and RPA is complemented towards BPMS. The positioning of RPA and RSO in comparison with BPMS is crucial. In analyzed case, there was mentioned about potential replacement of existing BPMS (K2) with RSO as the RPA robots will scale up.

- The biggest advantage of RPA is agility and flexibility provided to users with its short implementation cycles. It enables the RPA to quick scale up and to reach to the point where the robots have to be manageable inhouse - potential for RSO platform.

- Focus on process modelling and description is a key initial phase as the RPA is built on exact rules. The selection of process needs to focus on the ones precisely specified. The AI in RPA or RSO is still in its infancy and according to respondents, RPA tools are still rule-based.
Next few years will show if RPA and RSO are considered as a generally accepted tools for automating business processes and not only another package of existing technology, which is hyped by consulting and software houses. Further rigorous view has to be taken in order to reliably discover the benefits of this technology and how to achieve them.

\section{Acknowledgement}

This paper was supported by the SGS project of Silesian University in Opava, Czechia SGS/8/2018 "Advanced Methods and Procedures of Business Processes Improvement".

\section{Literature}

Aalst, W.M.P. van der, Bichler M. \& Heinzl A. (2018). Robotic Process Automation. Bus Inf Syst Eng, 60(4), 269-272, https://10.1007/s12599-018-0542-4

Abbott, K.R. \& Sarin, S.K. (1994). Experiences with Workflow Management: Issues for the Next Generation. In: Proceedings of the 1994 ACM Conference on Computer Supported Cooperative Work. (pp. 113-120). New York: ACM.

Aguirre, S. \& Rodriguez, A. (2017). Automation of a Business Process Using Robotic Process Automation (RPA): A Case Study. In Workshop on Engineering Applications, 27 September 2017 (pp. 65-71). Cham: Springer.

Asquith, A., Horsman, G. (2019). Let the robots do it! - Taking a look at Robotic Process Automation and its potential application in digital forensics. Forensic Science International: Reports, $\quad$ https://doi.org/10.1016/j.fsir.2019.100007

Dumas, M., Rosa, M.L., Mendling, J. \& Reijers, H. (2013). Fundamentals of Business Process Management. New York: Springer.

Feagin, J.R., Orum, A.M. \& Sjoberg, G. (1991). A Case for the Case Study. Chapel Hill: UNC Press Books.

Fersht, P. \& Slaby, R.J. (2012). Robotic automation emerges as a threat to traditionallow-costoutsourcing. Bangalore: HfS Research. Retrieved from https://www.horsesforsources.com/wp-content/uploads/2016/06/RS-1210 Robotic-automation-emerges-as-a-threat-060516.pdf

Fernandez, D. \& Aman, A. (2018). Impacts of Robotic Process Automation on Global Accounting Services. Asian Journal of Accounting and Governance, 9(1), 127140. http://dx.doi.org/10.17576/AJAG-2018-09-11

Hindle, J., Lacity, M., Willcocks, L. \& Khan, S. (2018). ROBOTIC PROCESS AUTOMATION: Benchmarking the ClientExperience.London:KnowledgeCapitalPartners.

Houy, C., Hamberg, M. \& Fettke, P. (2019). Robotic Process Automation in Public Administrations. Gesellschaft für Informatik e.V.

Lacity, M., Willcocks, L.P. \& Craig,A. (2015a). Robotic process automation: mature capabilities in the energy sec- 
tor. London: LSE Library. Retrieved from http://eprints. 1se.ac.uk/64520/1/OUWRPS 1506 published.pdf Lacity, M., Willcocks, L.P. \& Craig, A. (2015b). Robotic Process Automation at Telefónica O2. MIS Quarterly Executive, 15(1), 21-35. Lacity, M. \& Willcocks L.P. (2017). A new approach to automating services. London: LSE Library. Retrieved from http://eprints.lse.ac.uk/68135/1/ Wi1lcocks_New\%20approach_2016.pdf Lamberton, C. (2016). Get ready for robots: Why planning makes the difference between success and disappointment, London: Ernst \& Young. Retrieved from https://www.ey.com/Publication/vwLUAssets/Get ready_for_robots/\$FILE/ey-get-ready-for-robots.pdf Le Clair, C. (2017). The Forrester wave: robotic process automation: the 12 providers that matter most and how they stack up. Cambridge: Forrester. Retrieved from http://www.bluvaultsolutions. com/wp-content/uploads/2017/11/Robotics.pdf

Mendling, J., Decker, G., Hull, R., Reijers, H.A. \& Weber, I. (2018). How do Machine Learning, Robotic Process Automation, and Blockchains Affect the Human Factor in Business Process Management? Communications of the Association for Information Systems, 43, 297-320, https://doi.org/10.17705/1CAIS.04319

Michalos, G., Makris, S., Spiliotopoulos, J., Misios, I., Tsarouchi, P. \& Chryssolouris, G. (2014). ROBO-PARTNER: Seamless Human-Robot Cooperation for Intelligent, Flexible and Safe Operations in the Assembly Factories of the Future. Procedia CIRP, 23(1), 71-76, https://10.1016/j.procir.2014.10.079

Moffitt, K. C., Rozario, A. M., \& Vasarhelyi, M. A. (2018). Robotic Process Automation for Auditing. Journal of Emerging Technologies in Accounting, 15(1), 1-10. https://doi.org/10.2308/jeta-10589

Pellegrinelli, S., Moro, F. L., Pedrocchi, N., Molinari Tosatti, L. \& Tolio, T. (2016). A probabilistic approach to workspace sharing for human-robot cooperation in assembly tasks. CIRP Annals, 65(1), 57-60, https://10.1016/j.cirp.2016.04.035

Ranerup, A., Henriksen, H. Z. (2019). Value positions viewed through the lens of automated decision-making: The case of social services. Government Information Quarterly, https://doi.org/10.1016/j.giq.2019.05.004

Saunders, M., Lewis, P. \& Thornhill, A. (2016). Research Methods for Business Students $\left(7^{\text {th }}\right.$ ed.). Edinburgh: Pearson.

Schmitz, M., Dietze, C. \& Czarnecki, C. (2019). Enabling Digital Transformation Through Robotic Process Automation at Deutsche Telekom. In N. Urbach \& M. Röglinger (Eds.), Digitalization Cases: How Organizations Rethink Their Business for the Digital Age, Management for Professionals (pp. 15 - 33): Springer, https://doi.org/10.1007/978-3-319-95273-4 2

Schramm, W. (1971). Notes on Case Studies of Instructional Media Projects. Stanford: California Institute for Communication Research. Tornbohm, C., Dunie, R. (2017). Gartner mar- ket guide for robotic process automation software. Stamford: Gartner. Retrieved from https:// www.gartner.com/en/documents/3835771/market-guide-for-robotic-process-automation-software Vom Brocke, J., Maaß, W., Buxmann, P., Maedche, A., Leimeister, J. M. \& Pecht, G. (2018). Future Work and Enterprise Systems. Business \& Information Systems Engineering, 60(4), 357-366, https://10.1007/s12599-018-0544-2

Yin, R.K. (2014). Case Study Research: Design and Method (5th ed.). London: Sage.

Dalibor Šimek, PhD Student at the Department of Business Economics and Management at Silesian University in Opava, School of Business Administration in Karvina, Czech Republic. He is leading the project funded by Silesian University Grant System in area of Business Process Management. Research interests: Business Process Management, Robotic Process Automation, Process Improvement.

Roman Šperka, Associate Professor and Head of the Business Economics and Management Department at Silesian University in Opava, School of Business Administration in Karvina, Czech Republic. He has participated in several EU and institutional projects. He is Programme Co-Chair of the KES-AMSTA conference. Research interests: business process management, process mining, modelling and simulation of social systems. 